\title{
Ectopic Variceal Bleeding From Chronic Superior Mesenteric Vein Thrombosis After Hemorrhagic Pancreatitis
}

\author{
Kathryn Flynn ${ }^{1}$, Kevin Chung ${ }^{1}$, Thomas Brooke ${ }^{1}$, and Jonathan Keung ${ }^{1}$ \\ ${ }^{1}$ Walter Reed National Military Medical Center
}

November 11, 2021

\begin{abstract}
Varices are a common cause of gastrointestinal bleed, but a small percentage of these bleeds are due to ectopic varices. This case presents bleeding ectopic varices in the third part of the duodenum with portal hypertension secondary to chronic superior mesenteric vein thrombosis that developed after hemorrhagic pancreatitis.
\end{abstract}

\section{Ectopic Variceal Bleeding From Chronic Superior Mesenteric Vein Thrombosis After Hemor- rhagic Pancreatitis}

LT Kathryn Flynn, D.O. Walter Reed National Military Medical Center, Internal Medicine

k.flynn8820@gmail.com

COL (Ret.) Kevin Chung, M.D. Walter Reed National Military Medical Center, Pulmonary and Critical Care Medicine

kevin.chung@usuhs.edu

LT Thomas Brooke, M.D. Walter Reed National Military Medical Center, Internal Medicine

thomasrbrooke@gmail.com

MAJ Jonathan Keung, M.D. Walter Reed National Military Medical Center, Interventional Radiology

jonathan.j.keung.mil@mail.mil

Written informed consent was obtained from the patient to publish this report in accordance with the journal's patient consent policy.

\section{Abstract}

Intro

Varices are a common cause of gastrointestinal (GI) bleed. When ectopic, there is often a delay in diagnosis as it is difficult to localize these varices. Ectopic small bowel varices usually arise from portal hypertension, which commonly develops in the setting of cirrhosis. This case presents a much rarer cause of bleeding ectopic varices with portal hypertension secondary to chronic superior mesenteric vein (SMV) thrombosis that developed after an episode of hemorrhagic pancreatitis.

\section{Case Presentation}

An 81 year old male with a past medical history of a recent GI bleed secondary to an arteriovenous malformation presented to the hospital with continued melena after a recent admission at another hospital for the same symptom. Upper endoscopy and colonoscopy showed no evidence of active bleeding. Subsequent 
computed tomography angiography (CTA) showed bleeding from collaterals in the third part of the duodenum, consistent with ectopic varices. The CTA also showed SMV thrombosis. The patient underwent ultrasound-guided transhepatic venogram with coiling and sclerosant embolization of SMV varices and distal SMV balloon angioplasty. Capsule endoscopy after showed no evidence of further bleeding. The patient was discharged 72 hours after the intervention with a stabilized hemoglobin and resolved melena.

\section{Conclusions}

Ectopic varices should be on the differential diagnosis for patients presenting with a GI bleed that remains non-localized after endoscopy and colonoscopy. EGD or colonoscopy are the first line interventions for treatment of bleeding ectopic varices. If unreachable by these means, percutaneous coil embolization is an alternative way to stabilize the patient. As no general management guidelines exist, treatment of bleeding ectopic varices should continue to be case dependent and involve a multidisciplinary team.

\section{Keywords}

Gastroenterology, critical care, bleed, bleeding, gastrointestinal bleed, GI bleed, ectopic varices, varix, ectopic, pancreatitis, duodenum, duodenal, superior mesenteric vein thrombosis, interventional radiology, embolization, hemorrhagic pancreatitis, portal hypertension, endoscopy, colonoscopy, angioplasty, percutaneous coil embolization

\section{Introduction}

Varices are a common cause of gastrointestinal (GI) bleeds and are either gastroesophageal or ectopic. Ectopic varices, by definition, are large portosystemic venous collaterals located anywhere outside the gastroesophageal region. Ectopic varices account for only $5 \%$ of all variceal bleeds. Due to their infrequency and location outside the gastroesophageal region, ectopic variceal bleeds pose a diagnostic dilemma as these sources often take longer to localize in a patient presenting with a GI bleed ${ }^{1}$. Ectopic varices have a mortality rate of up to $40 \%$ during a bleeding episode and a four-fold increased risk of bleeding when compared to gastroesophageal varices ${ }^{1,2}$.

Ectopic varices most commonly develop in the setting of portal hypertension from pre-existing liver disease, such as cirrhosis. It is important to investigate other causes when a patient does not exhibit cirrhotic findings in the setting of portal hypertension. One cause of portal hypertension is superior mesenteric vein (SMV) thrombosis, and its most common presenting symptom is bleeding from esophageal or gastric varices ${ }^{3}$. The clinical presentation of bleeding ectopic varices from SMV thrombosis after hemorrhagic pancreatitis is rare to find in the literature. There is a 1-2\% reported incidence of portsosplenomesenteric thrombosis after severe acute pancreatitis ${ }^{4}$. To our knowledge, only two cases of bleeding small bowel varices after pancreatitis exist ${ }^{5}$. Here we present a case of bleeding duodenal varices caused by portal hypertension from SMV thrombosis secondary to hemorrhagic pancreatitis.

\section{Case Presentation}

An 81 year old male with a past medical history of a recent GI bleed presented to the hospital after two days of melena and left sided and mid-epigastric abdominal pain associated with fatigue and dizziness with ambulation. The patient had been discharged a week prior from a different intensive care unit (ICU) after a prolonged admission for a GI bleed. Colonoscopy during that admission showed descending colon arteriovenous malformation that was treated with argon plasma coagulation (APC) and ulcerated mucosa in the descending colon that was treated with epinephrine and clips. Esophagogastroduodenoscopy (EGD) showed evidence of Barrett's esophagus but no active bleeding. Both computed tomography angiography (CTA) and magnetic resonance imaging of the abdomen showed no evidence of active GI bleeding, but it was significant for chronic occlusion of the SMV and splenic vein with significant intramural/periduodenal venous collaterals. The patient was diagnosed with a lower GI bleed in the descending colon. The patient presented to this hospital shortly after discharge due to the continuation of melena and symptomatic anemia. Symptoms included feeling clammy, weak, and nauseated with dyspnea on exertion without chest pain. The patient reported continued black, tarry stools without pain during bowel movements. 
The patient was normotensive and without tachycardia. On physical exam, the patient was well-developed and in no apparent distress, but there was some mild tenderness to palpation on the mid-epigastrium and left lower quadrant. Hemoglobin was $8.1 \mathrm{~g} / \mathrm{dL}$ on admission, down from a baseline of $14 \mathrm{~g} / \mathrm{dL}$ two months prior. Liver function tests and coagulation studies (PT, PTT, INR) were within normal limits. The hemoglobin dropped to $6.9 \mathrm{~g} / \mathrm{dL}$ overnight, so the patient was admitted to the medical ICU with symptomatic anemia in the setting of an ongoing GI bleed.

EGD and colonoscopy both showed no active source of bleeding or targetable lesion. The patient received six units of RBCs while in the ICU. The hemoglobin stabilized with no source for an active bleed, so the patient was transferred to the general floor.

In the setting of known chronic SMV thrombosis, hematology/oncology performed a comprehensive hypercoagulable workup. The workup was completely negative and included JAK2 V617F Mutation, Lupus Anticoagulant, Direct Russell Viper Venom Time, Cardiolipin Antibodies, Beta-2 Glycoprotein Antibodies, Factor V Leiden, Factor II, and Protein C levels.

The patient was transferred back to the ICU two days later for symptomatic anemia with another hemoglobin drop to $5.8 \mathrm{~g} / \mathrm{dL}$. The patient received three more units of RBCs and had a CTA (Figure 1) that showed bleeding varices around the third part of the duodenum, which prompted the consultation of Interventional Radiology (IR) and General Surgery. IR performed a successful ultrasound-guided transhepatic venogram with coiling and sclerosant embolization of varices around the third part of the duodenum as seen in Figure 2 and Figure 3. Balloon angioplasty of distal SMV stenosis was also performed. After the procedure, Gastroenterology performed capsule endoscopy that showed no active bleeding. Subsequent CT imaging in Figure 4 showed resolution of the variceal network. The patient received a total of 12 units of RBCs during this admission. Fibroscan confirmed that the patient did not have cirrhosis. The patient's melena resolved, and his hemoglobin remained stable for 72 hours, at which point he was deemed safe to be discharged home.

At follow-up 1 year later, the patient has not had any recurrent bleeding episodes. He had a repeat EGD and colonoscopy without evidence of any bleeding.

\section{Discussion}

Patients with ectopic varices often have underlying portal hypertension in the setting of cirrhosis. SMV thrombosis is a known but uncommon cause of portal hypertension and represented $3 \%$ of reported cases in a study evaluating the etiology of portal hypertension in adults ${ }^{6}$. The increasing hydrostatic pressures from the backflow behind the thrombus can lead to bleeding varices, which most often form in the esophagus or stomach. Varices developing from SMV thrombosis can go undetected for long periods of time due to the vague, intermittent abdominal pain that may be the only symptom until a patient presents with bleeding collaterals. If EGD and colonoscopy cannot localize a bleeding source, the diagnosis becomes more difficult as the bleeding may be coming from ectopic varices ${ }^{3}$.

Ectopic varices, by nature of their location, are harder for clinicians to locate when a patient presents with a GI bleed as they can be unreachable by EGD and colonoscopy. The patient had undergone two prior endoscopies, but the endoscopies evaluated up to the second part of the duodenum and were just shy of the area of the bleeding varix. An interdisciplinary team was critical to identifying and treating the lesion, as the lesion was not identified until IR was consulted and found it on a personal read of the imaging. The prolonged hospital course and delayed diagnosis reinforces the relevant and challenging nature of ectopic varices.

Though chronic SMV thrombosis was identified on earlier imaging studies, this diagnosis does not immediately point clinicians towards suspecting bleeding duodenal varices because SMV thrombosis induced varices are often found in the esophagus or stomach. Chronic SMV thrombosis is often a sequela of prothrombotic states like coagulopathies or malignancy, local foci of irritation or inflammation like pancreatitis or splenectomy, or a combination of these. Any patient with SMV thrombosis should undergo a full hypercoagulable workup, and their history should be thoroughly examined for any precipitating events ${ }^{3}$. The most plausible 
cause of this patient's chronic SMV thrombosis was from an episode of hemorrhagic pancreatitis years ago, which led to a prothrombotic state of thrombosis formation and allowed for the gradual development of venous collaterals. A retrospective study from the University of Pittsburgh showed that 22 of 162 patients $(14 \%)$ with acute pancreatitis developed portosplenomesenteric venous thrombosis. Necrotizing pancreatitis was associated with all but one patient with thrombus formation, and $27 \%$ of these patients developed varices. All varices were localized to the gastroesophageal region, and no patients had any gastrointestinal bleeding complications ${ }^{7}$. While portosplenomesenteric venous thrombosis is a known complication of necrotizing pancreatitis, the majority of patients do not have long term sequelae of variceal bleeding or ectopic variceal formation ${ }^{4}$.

Due to the infrequency in which bleeding ectopic varices are identified, data regarding management is scarce and often comes from case reports and retrospective series ${ }^{3}$. In general, treatment requires a multidisciplinary approach and is tailored to each patient. After resuscitation and stabilization, the preferred intervention is endoscopic via EGD or colonoscopy. For bleeding ectopic varices unreachable by EGD or colonoscopy, percutaneous coil embolization is regarded as a safe short-term approach to stabilize the patient ${ }^{8}$. This patient successfully underwent this procedure based on the deliberation of the interventional radiologists and gastroenterologists regarding the most effective approach. Patients treated with embolization often have episodes of rebleeding and need a subsequent intervention ${ }^{1}$. More research is needed to better define prophylactic and therapeutic data when it comes to treating bleeding ectopic varices. One area of continued debate revolves around $\beta$-blocker secondary prophylaxis against duodenal variceal rebleeding, and more studies will need to be conducted to understand if and who this treatment would most benefit ${ }^{1}$. Chronic SMV thrombosis is often treated with long-term anticoagulation, but large studies have not evaluated the risks and benefits of anticoagulation in the setting of bleeding varices ${ }^{3}$. As no general management guidelines exist, treatment of bleeding ectopic varices should continue to be case dependent and involve a multidisciplinary team.

\section{Conclusions}

In any patient with portal hypertension and GI bleeding, the presence of ectopic varices must be considered if both EGD and colonoscopy fail to localize a bleeding source. This patient's remote history of hemorrhagic pancreatitis allowed for a hypercoagulable condition in which chronic SMV thrombosis developed, thus propagating undiagnosed portal hypertension until presenting with ectopic variceal bleeding. EGD or colonoscopy are the first line interventions for treatment of bleeding ectopic varices. If unreachable by these means, percutaneous coil embolization is an alternative way to stabilize the patient. As no general management guidelines exist, treatment of bleeding ectopic varices should continue to be case dependent and involve a multidisciplinary team.

No competing interests to declare.

Kathryn Flynn: led the writing, editing, revising and followed up the patient; Thomas Brooke and Kevin Chung: contributed to the clinical presentation of the patient and helped with revisions; Jonathan Keung: contributed to the imaging findings and interpretations.

The authors declare that they have no conflicts of interest and no funding was received for this case report. No patient identifying data has been released in the article. No further acknowledgements to be made.

\section{References}

1. Helmy A, Al Kahtani K, Al FM. Updates in the pathogenesis, diagnosis and management of ectopic varices. Hepatol Int. 2008;2(3):322-34. doi: 10.1007/s12072-008-9074-1.

2. Kochar N, Tripathi D, McAvoy NC, Ireland H, Redhead DN, Hayes PC. Bleeding ectopic varices in cirrhosis: The role of transjugular intrahepatic portosystemic stent shunts. Aliment Pharmacol Ther. 2008;28:294303.

3. Harnik IG, Brandt LJ.Mesenteric venous thrombosis.Vasc Med. 2010; 15:407-418. doi: 


\subsection{7/1358863X10379673.}

4. Gonzelez HJ, Sahay SJ, Samadi B, Davidson BR, Rahman SH. Splanchnic vein thrombosis in severe acute pancreatitis: a 2-year, single-institution experience. HPB (Oxford) 2011;13(12):860-864. doi: 10.1111/j.1477-2574.2011.00392.x.

5. Bommana V, Shah P, Kometa M, Narwal R, Sharma P. A case of isolated duodenal varices secondary to chronic pancreatitis with review of literature. Gastroenterology Res. 2010;3:281-6.

6. Goel A, Madhu K, Zachariah U, Sajith KG, Ramachandran J, Ramakrishna B, Gibikote S, Jude J, Chandy GM, Elias E, Eapen CE. A study of aetiology of portal hypertension in adults (including the elderly) at a tertiary centre in southern India. Indian J Med Res. 2013;137:922-927.

7. Easler J, Muddana V, Furlan A, et al. Portosplenomesenteric venous thrombosis in patients with acute pancreatitis is associated with pancreatic necrosis and usually has a benign course. Clin Gastroenterol Hepatol. 2014;12:854-862.

8. Sarin SK, Kumar CKN. Ectopic varices. Clin Liver Dis (Hoboken) 2012;1:167-172.

Bhat A, Davis R, Bryan W. A rare case of bleeding duodenal varices from superior mesenteric vein obstruction-treated with transhepatic recanalization and stent placement. Indian J Radiol Imaging. 2019;29:313. doi: 10.4103/ijri.IJRI_21_19.

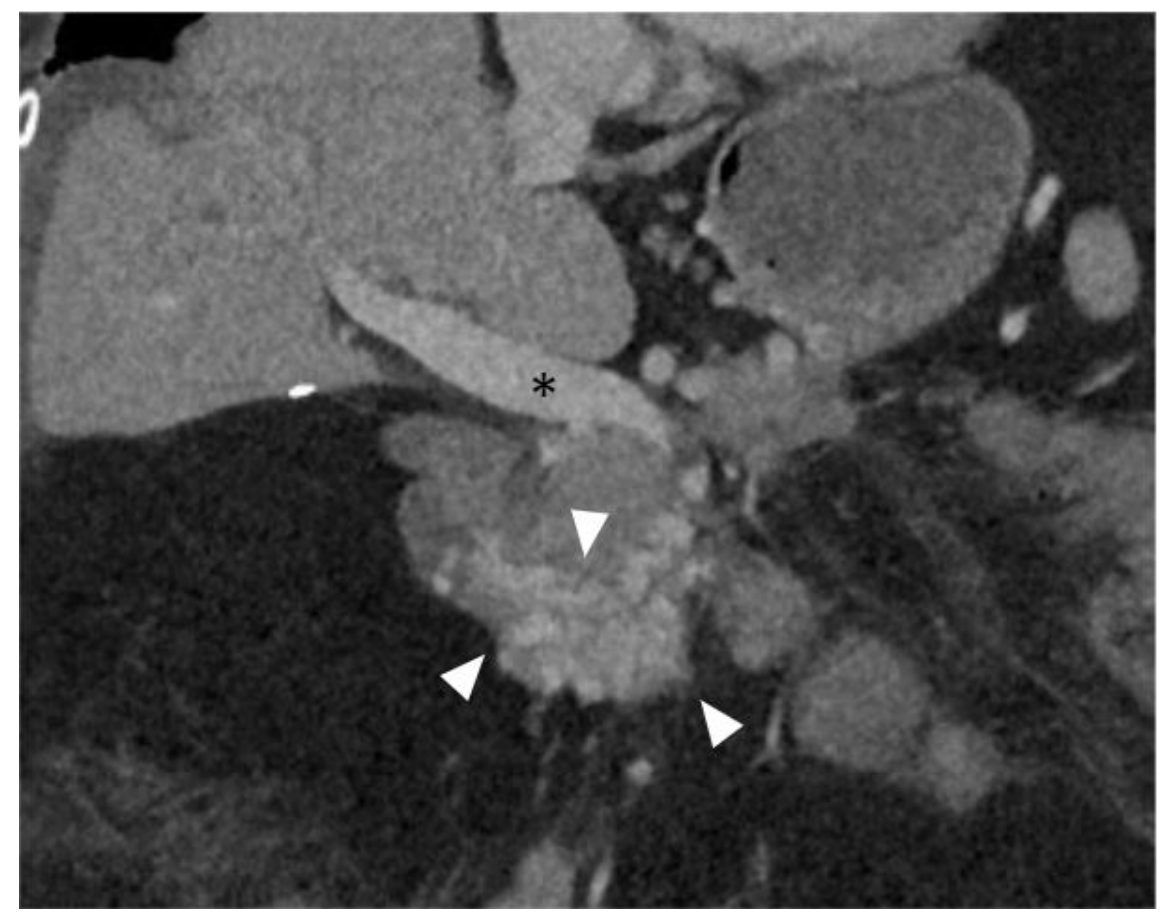



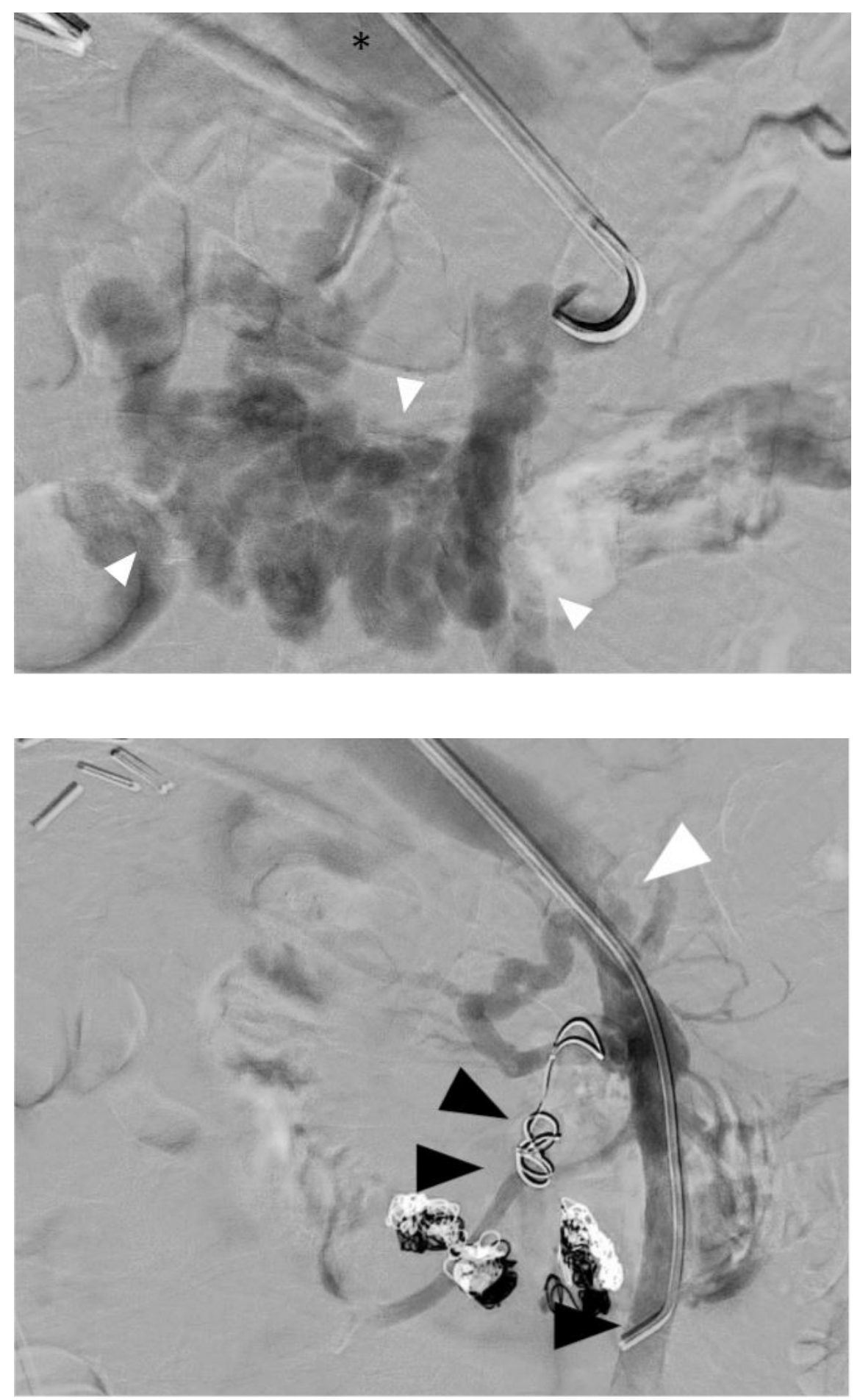


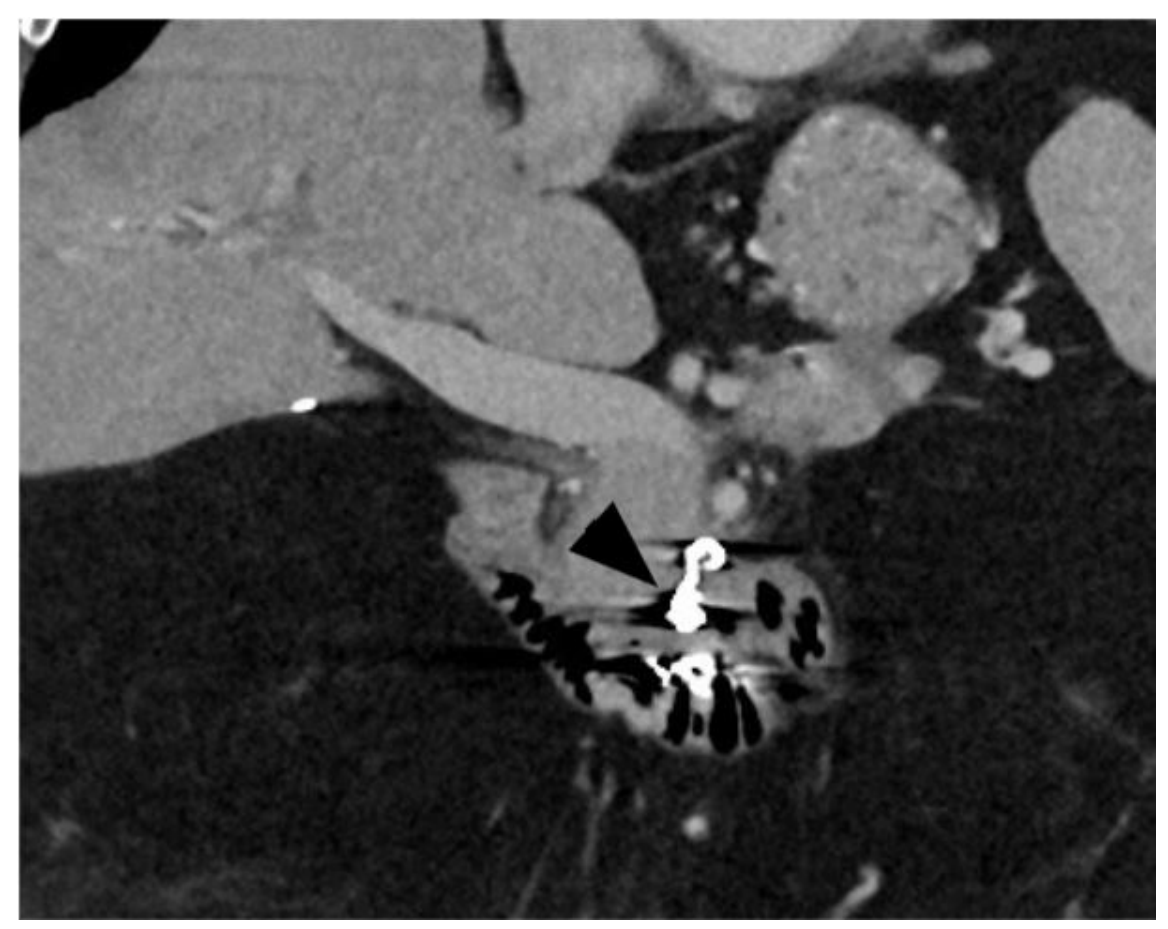

